\title{
Palatability and physical properties of potassium- binding resin RDX7675: comparison with sodium polystyrene sulfonate
}

This article was published in the following Dove Press journal:

Drug Design, Development and Therapy

6 September 2017

Number of times this article has been viewed

\author{
Vanessa Zann' \\ John McDermott ${ }^{\prime}$ \\ Jeffrey W Jacobs ${ }^{2}$ \\ James P Davidson ${ }^{2}$ \\ Fangling Lin $^{2}$ \\ Paul Korner ${ }^{2}$ \\ Robert C Blanks ${ }^{2}$ \\ David P Rosenbaum ${ }^{2}$ \\ 'Quotient Clinical, Nottingham, UK; \\ ${ }^{2}$ Ardelyx Inc., Fremont, CA, USA
}

Correspondence: David P Rosenbaum Ardelyx Inc., 34175 Ardenwood Boulevard, Fremont, CA 94555, USA

Tel +l 5107451752

Email drosenbaum@ardelyx.com
Background: Hyperkalemia is a potentially life-threatening condition that patients with heart failure or chronic kidney disease, especially those taking renin-angiotensin-aldosterone system inhibitors, are at high risk of developing. Sodium polystyrene sulfonate (SPS), a current treatment, binds potassium within the gastrointestinal tract to reduce potassium absorption. However, poor palatability limits its long-term use. RDX7675, a novel potassium binder in development for the treatment of hyperkalemia, is a calcium salt of a reengineered polystyrene sulfonate-based resin designed to have enhanced palatability. Here, the physical properties and palatability of RDX7675 and SPS are compared.

Methods: RDX7675 and SPS particle sizes were measured using wet dispersion laser diffraction. Palatability was assessed in a randomized, crossover, healthy volunteer study with two visits. At visit 1 (open label), volunteers evaluated high-viscosity, intermediate-viscosity, and waterreconstituted formulations of RDX7675 (all vanilla flavor), and an equivalent reconstituted SPS (Resonium $\mathrm{A}^{\circledR}$ ). At visit 2 (single-blind), volunteers evaluated RDX7675 as a high-viscosity formulation in vanilla, citrus, and mint flavors, and as intermediate-viscosity, low-viscosity, and reconstituted formulations in citrus flavor. Volunteers used a "sip and spit" technique to rate overall acceptability and seven individual characteristics from 1 ("dislike everything") to 9 ("like extremely").

Results: RDX7675 particles were smaller than SPS particles, with a narrower size range (RDX7675, 80\%, 14-52 $\mu \mathrm{m}$; SPS, 11.3-124.2 $\mu \mathrm{m}$ ), and had a smooth, spherical shape, in contrast to the shard-like SPS particles. Reconstituted RDX7675 was considered superior to SPS for five of the seven palatability characteristics and for overall acceptability (median, visit 1: reconstituted RDX7675, 5.0; SPS, 4.0). High-viscosity vanilla was the most highly rated RDX7675 formulation (median overall acceptability, visit 2: 7.0).

Conclusion: The smaller, more uniformly shaped, spherical particles of RDX7675 resulted in improved palatability over SPS when reconstituted in water. The overall results are promising for future patient acceptability of RDX7675 treatment.

Keywords: hyperkalemia, potassium, heart failure, chronic kidney disease, adherence, drug formulation

\section{Introduction}

Hyperkalemia, typically defined as a serum potassium level greater than 5.0 or $5.5 \mathrm{mEq} / \mathrm{L}$, is a relatively common and clinically relevant problem in patients with cardiovascular and renal diseases that accounts for $3 \%$ of the US hospitalizations. ${ }^{1,2}$ Hyperkalemia has the potential to lead to life-threatening cardiac arrhythmias, and studies have shown that elevated potassium levels increase the risk of death. ${ }^{3-6}$ 
Conditions such as heart failure and chronic kidney disease (CKD) are associated with a significantly higher risk of hyperkalemia, ${ }^{3,6,7}$ and serum potassium levels greater than $5.0 \mathrm{mEq} / \mathrm{L}$ are reported in more than half of predialysis patients with CKD. ${ }^{8}$ Hyperkalemia is also commonly associated with the use of some medications. ${ }^{9,10}$ Renin-angiotensinaldosterone system (RAAS) inhibitors are recommended by evidence-based treatment guidelines for the reduction of morbidity and mortality in both patients with heart failure and individuals with CKD. ${ }^{11-13}$ However, these medications can decrease potassium excretion, leading to an increased frequency of hyperkalemia..$^{9,14,15}$ Despite the benefits of RAAS inhibition therapy, when hyperkalemia occurs, cardiologists and nephrologists follow current guideline recommendations that dosing of these agents be reduced or discontinued. ${ }^{11-13,16}$ Therefore, the concomitant long-term use of a serum potassium-lowering medication could facilitate optimal RAAS inhibitor therapy in this patient group.

The dominant mode of treatment for hyperkalemia is oral administration of cation exchange resins. These insoluble materials sequester excess potassium cations in the gastrointestinal tract, preventing them from being absorbed and leading to their elimination in the feces. ${ }^{17}$ Sodium polystyrene sulfonate (SPS; trade names Kayexalate ${ }^{\circledR}$ [Sanofi US, Bridgewater, NJ, USA] or Resonium $A^{\circledR}$ [Sanofi Winthrop Industrie, Amilly, France] - the active ingredient in the latter is chemically identical to Kayexalate, but the brand is not marketed in the US) is an insoluble ion exchange resin that has been available since the 1950 s and is a mainstay of hyperkalemia treatment. ${ }^{17}$ There are, however, limitations associated with SPS that restrict its suitability for long-term use in patients with recurrent or chronic hyperkalemia. SPS is a milled product and consists of large, hard, irregularly shaped shardlike particles that give it a gritty texture in the mouth which, together with its unpleasant taste, reduces palatability and limits its use as a long-term treatment option. As described above, the risk of recurrent or chronic hyperkalemia is increased in individuals with heart failure and/or CKD, especially those taking RAAS inhibitors. These patients are prescribed multiple medications to take at various times of the day, making acceptability of a treatment particularly important to facilitate its long-term use. Furthermore, SPS contains sodium counter exchange ions; its use may therefore blunt the patient response to RAAS blockade and counteract the dietary salt restriction recommended for these individuals. ${ }^{11,13,18}$ Each gram of SPS taken removes $0.5-1.0 \mathrm{mmol}$ of potassium in exchange for 2-3 mmol of sodium; therefore, its use is associated with significant sodium infusion and can result in volume overload in susceptible populations. ${ }^{19}$ Finally, SPS is often coadministered with sorbitol to alleviate its constipating effect, but this may be associated with gastrointestinal adverse events. ${ }^{20}$

RDX7675, a reengineered polystyrene sulfonate with a calcium counter exchange ion, is a novel potassium-binding resin being developed for the treatment of patients with hyperkalemia. RDX7675 has been specifically designed to avoid the undesirable aspects of SPS, with the goal of obtaining an agent consisting of small, smooth, and malleable particles that translate into improved palatability. The use of calcium rather than sodium as the counter ion is aligned with best clinical practice for patients with heart failure and/or CKD, ${ }^{11,13}$ and it is not intended to be taken with sorbitol. Such an agent may be suitable for long-term management of hyperkalemia in patients with heart failure and/or CKD taking RAAS inhibitors. Here, the physical properties of RDX7675 and SPS are compared, and their relative palatability in healthy volunteers is examined. The effects of different formulations and flavors of RDX7675 on its palatability are also assessed.

\section{Methods}

\section{Structure and synthesis of RDX7675}

RDX7675 is the calcium salt of a sulfonated copolymer of divinylbenzene and styrene, commonly known as polystyrene sulfonate (Figure 1). It is an ionic, cross-linked copolymer that is insoluble in water and organic solvents. RDX7675 is prepared as small, spherical beads via the copolymerization of styrene and divinylbenzene in an aqueous suspension, with the divinylbenzene monomer added to achieve a nominal $1.8 \%$ cross-linked polystyrene polymer. This intermediate is sulfonated, washed, and then treated with a basic form of calcium to produce the desired calcium salt.

\section{Determination of particle size distribution and swelling ratio of RDX7675 and SPS}

Particle size was measured by wet dispersion laser diffraction using a Mastersizer 2000 (Malvern Instruments, Malvern,

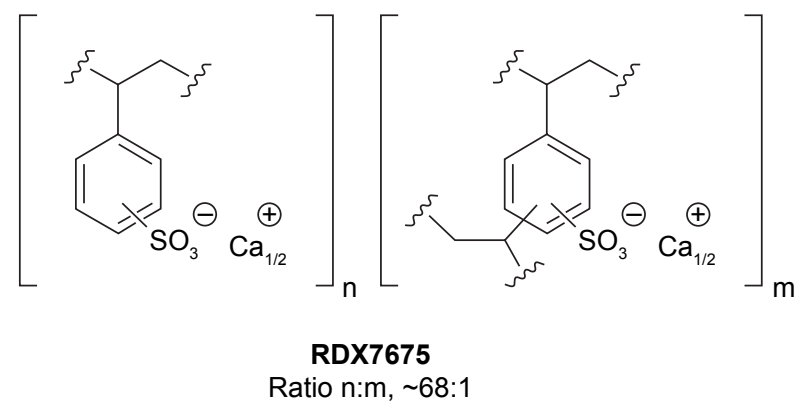

Figure I Chemical structure of RDX7675. 
UK). Samples were introduced as suspensions in deionized water into a Hydro 2000S sampler (Malvern Instruments), sonicated if necessary to break down agglomeration, and allowed 5-10 minutes of circulation for equilibration before measurements were taken.

The swelling ratio was measured using a centrifugation method. Approximately $1 \mathrm{~g}$ of calcium polystyrene sulfonate resin was weighed and then immersed in approximately $10-15 \mathrm{~mL}$ of deionized water (or $0.9 \%$ saline solution) in a $50-\mathrm{mL}$ preweighed centrifuge tube, and shaken for a minimum of 30 minutes. The tube was then spun at a relative centrifugal force of $2,000 \times g$ or $2,500 \times g$ for 30 minutes, and the supernatant was removed. The weight of the pellet (ie, the wet sample) was determined, and the ratio between the wet sample weight and the dry sample weight was calculated.

\section{RDX7675 formulation design}

Several formulations of different viscosities in which to suspend the RDX7675 beads were developed for palatability assessment in healthy volunteers. Based on measurements performed on commercial liquid food products, including fruit smoothies and syrups, formulations with a range of viscosities from drinkable liquids to spoonable semisolids were defined. These were labeled "low-viscosity" (resembling a yogurt), "intermediate-viscosity", and "highviscosity" (resembling a thick jelly) formulations according to their consistency, and were prepared using RDX7675 in water thickened with increasing quantities of xanthan gum $(0.33 \%, 0.67 \%$, and $1.0 \%$, respectively). These formulations, and a water-reconstituted formulation of RDX7675 (prepared without xanthan gum in the same way as SPS), were prepared for comparison with reconstituted SPS. To enhance the palatability of RDX7675 further, vanilla, citrus, and wintergreen garden mint flavors of the agent were also evaluated in this study.

\section{Palatability of RDX7675 in healthy volunteers}

The palatability, in terms of taste and mouth feel, of the different RDX7675 formulations was evaluated in a Phase I, single-center, randomized crossover study in healthy volunteers. The study was conducted in accordance with the International Conference on Harmonisation Good Clinical Practice guidelines approved by the Committee for Medicinal Products for Human Use and the ethical principles outlined in the World Medical Association Declaration of Helsinki and its amendments. The study protocol and its amendments were approved by the Wales Research Ethics Committee 1 (Health and Care Research Wales, Cardiff, UK), and all participants provided written informed consent before entering the study.

\section{Study design}

Palatability was assessed at two study visits separated by an interval of approximately 14 days, which allowed taste assessment data from visit 1 to be reviewed before selection of the formulations to be evaluated at visit 2. At visit 1 , volunteers tested four different formulations; at visit 2 , six different formulations were tested. Healthy volunteers were screened for inclusion in the study up to 28 days before the first visit. At each visit, eligible participants were admitted to the study unit at approximately 21:00 on the evening before the assessment day. On the study day, volunteers performed the first taste test approximately 2 hours after a standard breakfast. There was a washout period of at least 1 hour between each taste test, during which time participants were allowed to cleanse their palates using mineral water and unsalted crackers. Apart from this, participants fasted from all food and drink, and were not permitted to use chewing gum, until completion of the taste tests.

\section{Participants}

Healthy men and women aged 18 years or older with a body mass index of $18.0-35.0 \mathrm{~kg} / \mathrm{m}^{2}$ were eligible for inclusion in the study. Women of childbearing potential had to agree to use an appropriate method of contraception; women who were pregnant or lactating were excluded. Key exclusion criteria were as follows: a medical history that could impair their ability to taste; a clinically significant medical history or laboratory finding that could increase the risk to the participant, such as hypokalemia, hypocalcemia, obstructive bowel disease, or diseases that might cause difficulty swallowing; a history of any drug or alcohol abuse in the past 2 years; regular alcohol consumption exceeding 21 units/week in men and 14 units/week in women; and current smoking or smoking within the past 12 months. Volunteers who had taken any prescribed or over-the-counter drug (other than up to $4 \mathrm{~g}$ /day paracetamol, hormone replacement therapy, and hormonal contraception), herbal remedies, or experimental medication in the 14 days before the first day of the taste tests were also excluded.

\section{Test formulations}

At the first study visit, participants tasted each of the following products in a random order:

- SPS (Resonium A) $1 \mathrm{~g}$, reconstituted in water in line with the patient instructions on the package insert, vanilla flavor 
- RDX7675 $1 \mathrm{~g}$ as a reconstituted formulation prepared in the same way as SPS (ie, in water, with added sweetener and vanilla flavor to match the SPS formulation)

- RDX7675 $1 \mathrm{~g}$ as an intermediate-viscosity formulation (0.67\% xanthan gum), vanilla flavor

- RDX7675 $1 \mathrm{~g}$ as a high-viscosity formulation (1.0\% xanthan gum), vanilla flavor.

After the first study visit, an interim data review was conducted to select the RDX7675 formulations to be tasted at the second study visit:

- High-viscosity formulation (1.0\% xanthan gum), vanilla flavor

- High-viscosity formulation (1.0\% xanthan gum), citrus flavor

- High-viscosity formulation (1.0\% xanthan gum), wintergreen garden mint flavor

- Intermediate-viscosity formulation ( $0.67 \%$ xanthan gum), citrus flavor

- Low-viscosity formulation ( $0.33 \%$ xanthan gum), citrus flavor

- Reconstituted formulation (no xanthan gum), citrus flavor.

All formulations were administered orally in a volume of 4-6 mL. The quantity of resin at visit 2 varied within the volume, but was no more than $2 \mathrm{~g}$ for any regimen. Each volunteer received the formulations in a random order at visit 2, apart from the reconstituted RDX7675 formulation, which was always administered last. Treatment allocation sequences were determined using a Latin square design, and participants were assigned to a sequence using a computergenerated randomization schedule, with numbers balanced for each allocation sequence. Testing at visit 1 was openlabel, while that at visit 2 was single-blind (except for the reconstituted RDX7675 formulation).

\section{Taste test and evaluation}

A standardized "sip and spit" technique was followed for each taste test, in which participants were asked to rinse out their mouth with tap water, sip the sample from the spoon and roll it around their mouth for 3 seconds before spitting it out, and then rinse out their mouth with water. Participants were trained in the technique before the first taste test and were observed by study staff during testing to ensure compliance.

Immediately after each test, volunteers completed a validated questionnaire (developed by Sensory Research Ltd, Cork, Ireland) rating the acceptability of seven characteristics (smell, sweetness, bitterness, flavor, mouth feel/texture, grittiness, and aftertaste), as well as overall acceptability, on a 9-point scale ranging from 1 (“dislike everything") to 9 ("like extremely").

\section{Data analysis}

The taste assessment population comprised all volunteers who received at least one formulation of RDX7675 or SPS. Data from the taste test were summarized by regimen for visit 1 and visit 2 separately. Based on experience from previous similar studies, a total of 20 enrolled volunteers (expected to result in a minimum of 16 evaluable volunteers) was considered to be sufficient for this exploratory study.

\section{Results}

\section{Physical properties of RDX7675 and comparison with SPS}

RDX7675 had the appearance of a cream to brown powder consisting of malleable spherical beads with a median particle size of approximately $30 \mu \mathrm{m}$ and with $80 \%$ of particles in the range $14-52 \mu \mathrm{m}$, as measured using a wet dispersion technique (Table 1). By contrast, SPS had a wider range of particle sizes when measured using the same technique (Table 1). The appearance of the smooth, spherical RDX7675 beads contrasted with the irregularly shaped, shard-like particles of SPS (Figure 2). RDX7675 contained 8.5\%-11.5\% weight per weight of calcium, calculated on an anhydrous basis, and less than $0.1 \%$ of sodium. The swelling ratio of RDX7675 in water averaged 5.2, compared with 2.9 for SPS (Table 1).

\section{RDX7675 human palatability study}

In total, 20 healthy volunteers ( 8 men and 12 women) were enrolled in the RDX7675 formulation palatability study.

Table I Key physical and chemical properties of RDX7675 and SPS

\begin{tabular}{|c|c|c|c|}
\hline Parameter & RDX7675 & $\begin{array}{l}\text { SPS: } \\
\text { Kayexalate }^{\circledR}\end{array}$ & $\begin{array}{l}\text { SPS: } \\
\text { Resonium } A^{\circledR}\end{array}$ \\
\hline $\begin{array}{l}\text { Calcium content } \\
(\%, w / w)^{\mathrm{a}}\end{array}$ & $8.5-11.5$ & 0.001 & 0.011 \\
\hline $\begin{array}{l}\text { Sodium content } \\
(\%, w / w)^{\mathrm{a}}\end{array}$ & $\leq 0.1$ & 11.6 & 10.9 \\
\hline Water content (\%) & $\leq 10.0$ & 6.6 & 8.7 \\
\hline Particle size $(\mu \mathrm{m})$ & & & \\
\hline$D_{10}$ & $14.4 \pm 1.2$ & 8.2 & 11.3 \\
\hline$D_{50}$ & $31.0 \pm 1.3$ & 45.9 & 55.3 \\
\hline$D_{90}$ & $52.2 \pm 1.7$ & 119.7 & 124.2 \\
\hline Swelling ratio (water) & 5.2 & 2.9 & 2.9 \\
\hline
\end{tabular}

Notes: $\mathrm{D}_{\mathrm{x}}$ represents distribution ${ }_{\left.(\times)_{0}\right)}{ }^{\mathrm{a}} \mathrm{C}$ alculated on an anhydrous basis. ${ }^{\mathrm{b}}$ Measured using a wet dispersion technique. RDX7675 data are mean \pm SD values obtained from eight different batches of drug substance; data for Kayexalate ${ }^{\circledR}$ and Resonium $A^{\circledast}$ are from a single batch of each drug.

Abbreviations: SPS, sodium polystyrene sulfonate; SD, standard deviation; w/w, weight per weight. 

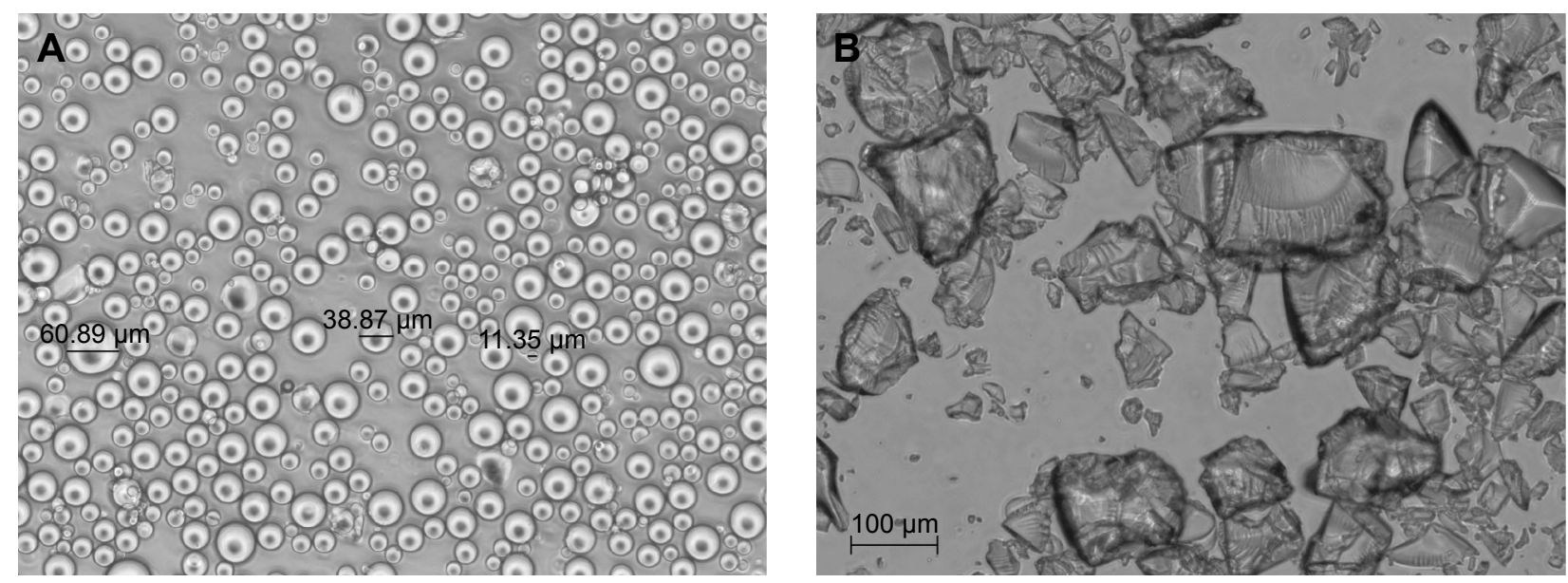

Figure 2 Optical micrograph of RDX7675 (A) and sodium polystyrene sulfonate (B; Kayexalate ${ }^{\circledR}$ ) particles in water, at the same magnification.

Nineteen volunteers were white, and one was Asian. Their ages ranged from 19 to 65 years (mean \pm standard deviation [SD]: $35.9 \pm 15.4$ years), and their mean $( \pm \mathrm{SD})$ body mass index was $25.0( \pm 3.2) \mathrm{kg} / \mathrm{m}^{2}$. All volunteers completed all of the taste tests at visits 1 and 2 .

\section{Visit I}

A summary of the taste and overall acceptability assessments at visit 1 is given in Table 2 and Figure 3. SPS had the lowest overall acceptability among the formulations tested at visit 1 (median score: 4.0; Table 2), with eight of the 20 participants ( $40 \%$ ) rating it as 2 ("dislike very much") or 3 ("dislike moderately") (Figure 3). The equivalent formulation of RDX7675, reconstituted with vanilla flavor, scored more highly than SPS (median overall acceptability score: 5.0). In total, nine participants (45\%) gave the RDX7675 reconstituted formulation a positive rating for overall acceptability of 6 ("like slightly") to 9 ("like extremely"), compared with four (20\%) for SPS. SPS also had the lowest or equal lowest median scores for all of the individual characteristics, and was particularly poorly rated for mouth feel/ texture (median: 3.0) and grittiness (median: 3.0) (Table 2). For mouth feel/texture, scores ranged from 2 ("dislike very much") to 6 ("like slightly"), with 17 of the 20 participants (85\%) rating it as 4 or below. For grittiness, scores ranged from 1 ("dislike everything") to 5 ("neither like nor dislike"), with 18 of the 20 participants (90\%) rating it as 4 ("dislike slightly") or below. The RDX7675 reconstituted formulation was rated more highly than SPS for five of the seven individual assessment items, and was rated equal for the remaining two. Of note, for the RDX7675 reconstituted formulation, the median score for mouth feel/texture was 4.5 (vs 3.0 for SPS), and for grittiness, it was 3.5 (vs 3.0 for SPS).
Among the RDX7675 formulations of various viscosities, the high-viscosity vanilla formulation had the highest median score (7.0) for overall acceptability of the four formulations tested at visit 1 . In all, 13 of the 20 participants (65\%) gave this formulation a score of 7 ("like moderately") or above, with scores ranging from 3 ("dislike moderately") to 8 ("like very much"). The RDX7675 intermediate-viscosity vanilla formulation also had a high rating for overall acceptability (median: 6.0), with scores ranging from 4 ("dislike slightly") to 8 ("like very much"). The RDX7675 high-viscosity vanilla and intermediate-viscosity vanilla formulations also scored most highly for the individual assessment items (Table 2).

\section{Visit 2}

On the basis of the results from visit 1, the RDX7675 high-viscosity vanilla formulation was considered to have the most acceptable taste profile and mouth feel, and was therefore selected for further assessment at the second visit. Two other flavors of the high-viscosity formulation, citrus and mint, were also tested at visit 2 . Testing of the RDX7675 intermediate-viscosity vanilla formulation produced similar results to the high-viscosity formulation tested at visit 1 , so the intermediate-viscosity formulation underwent further testing at visit 2 in another flavor, citrus. In addition, a yogurtlike formulation with lower viscosity than the previously tested high and intermediate viscosities, and a reconstituted formulation, both citrus flavor, were tested at visit 2 .

A summary of the taste and overall acceptability assessments at visit 2 is given in Table 3 and Figure 4. Among the citrus-flavored formulations tested at visit 2 , the highviscosity formulation was preferred over the formulations of lower viscosity, having the joint highest median overall acceptability score of all formulations tested (7.0; Table 3). 


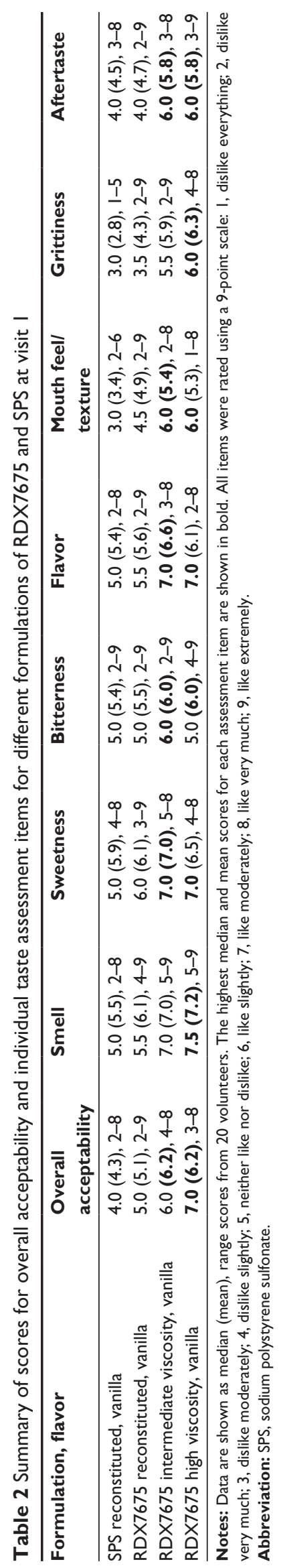

The reconstituted formulation in citrus flavor was the least acceptable of the formulations tested at visit 2 (median score: 4.0$)$, with 14 of the $20(70 \%)$ participants scoring it as 4 ("dislike slightly") or below (Figure 4). While the RDX7675 high-viscosity formulations in vanilla and citrus flavors both had equal median scores for overall acceptability (7.0), vanilla had the higher mean score of the two flavors (6.8 vs 6.4). Compared with the citrus-flavored high-viscosity formulation, the vanilla-flavored high-viscosity formulation had a narrower range of scores, with 17 of the 20 participants (85\%) rating it as 6 ("like slightly") or above (Figure 4).

The RDX7675 high-viscosity vanilla and citrus formulations scored most highly for the individual assessment items (Table 3), with vanilla having the highest or joint highest median and mean scores across all items apart from grittiness. For the high-viscosity vanilla formulation, 4 ("dislike slightly") was the lowest rating from any participant for any of the seven measures, and at least 18 of the 20 participants (90\%) rated it as 5 ("neither like nor dislike") or above for all items except grittiness. The reconstituted formulation had the lowest or joint lowest median scores for all of the individual items. Median scores for the reconstituted formulation were lowest for mouth feel/texture, grittiness, and aftertaste (each 4.0 ), with 14 of the 20 participants (70\%) rating the reconstituted formulation as 4 ("dislike slightly") or below for mouth feel/texture and grittiness, and 11 of the 20 participants (55\%) rating it as 4 or below for aftertaste.

\section{Discussion}

RDX7675 is a novel potassium-binding resin being developed for the treatment of patients with hyperkalemia. It is a polystyrene sulfonate resin with a calcium counter exchange ion, which binds potassium ions in the gut by exchanging the counter ion for potassium, thereby reducing potassium absorption. Patients with heart failure and/or CKD, especially those taking RAAS inhibitors, are at high risk of developing hyperkalemia. ${ }^{9}{ }^{14}$ Concomitant administration of a serum potassium-lowering therapy that is acceptable to patients for long-term use would facilitate receipt of the maximum benefit from an optimized RAAS inhibitor regimen, as recommended by treatment guidelines. ${ }^{11-13} \mathrm{RDX} 7675$ has been designed to have optimum palatability, with the goal of obtaining an agent suitable for long-term management of hyperkalemia.

Current polystyrene sulfonate-based potassium exchange resins consist of large, hard, and irregular shard-like particles. This gives them sand-like properties in the mouth, which may have a negative impact on patient acceptability and adherence to treatment. SPS, for example, is a milled product that 


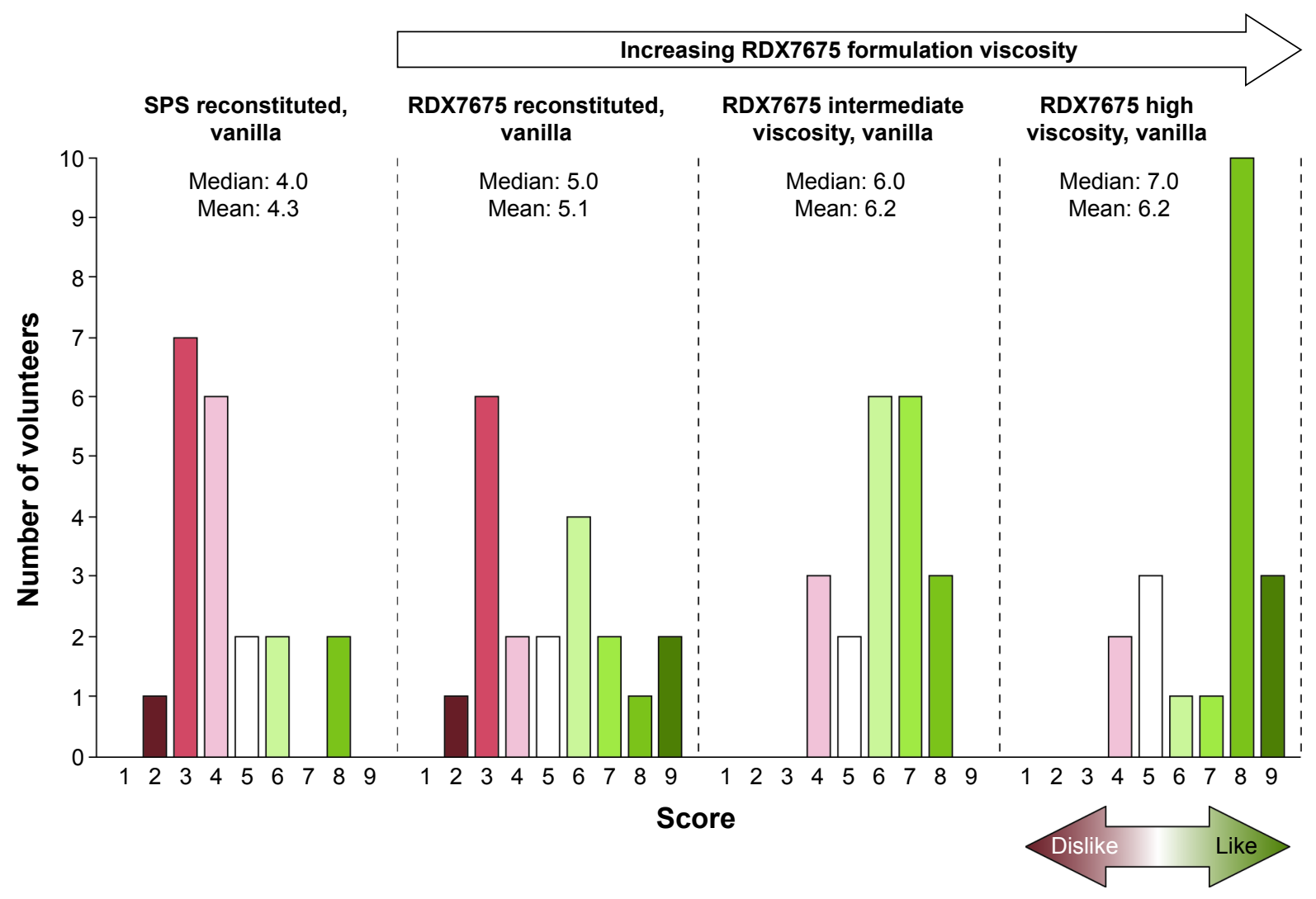

Figure 3 Distribution of scores for overall acceptability of different formulations of RDX7675 and sodium polystyrene sulfonate at visit I.

Notes: Each formulation was rated by 20 healthy volunteers. Overall acceptability was rated on a 9-point scale (I, dislike everything; 2 , dislike very much; 3 , dislike moderately; 4, dislike slightly; 5, neither like nor dislike; 6, like slightly; 7, like moderately; 8, like very much; 9, like extremely); no participant gave any formulation a score of I. Abbreviation: SPS, sodium polystyrene sulfonate.

consists of irregularly shaped particles with a large range of sizes; in the present study, $80 \%$ of the particles were found to have a diameter in the range $\sim 10-120 \mu \mathrm{m}$. Other investigators report that SPS particles have an average diameter of $\sim 165 \mu \mathrm{m}$ (measured dry) (1) $^{21}$ or $<150 \mu \mathrm{m}$ (measured wet). ${ }^{22}$ In contrast, the RDX7675 polystyrene sulfonate resin has been reengineered to have a low level of cross-linking, leading to the generation of more malleable and uniformly sized particles. Here, RDX7675 was confirmed to have a relatively narrow size range, with $80 \%$ of particle diameters in the range $14-52 \mu \mathrm{m}$. The spherical shape of the RDX7675 particles, in contrast to the shard-like SPS particles, and their higher swelling ratio compared with SPS may contribute to RDX7675 having a smoother, softer feel in the mouth. Subsequently, in the study in healthy volunteers comparing the palatability of RDX7675 with SPS, the latter had the lowest or equal lowest median scores for all of the individual taste assessment items, and received particularly low ratings for mouth feel/texture and grittiness. Furthermore, SPS was rated lowest for overall acceptability, with the majority of participants disliking it "slightly", "moderately", or "very much". In contrast, the water-reconstituted formulation of RDX7675 was rated higher than SPS, both overall and for five of the seven individual items, even though the two formulations were prepared in the same way. This shows that the underlying reengineered physical properties of RDX7675 described here have a marked positive effect on its palatability.

In addition to the physical properties of the particles, the formulation of an ion exchange resin is important for its palatability. As well as flavor, a key characteristic considered during formulation development is the viscosity of the substance in which the particles are suspended, which can further attenuate the sensation of foreign matter on the palate. Several RDX7675 formulations of differing viscosities were therefore evaluated in this study: all of the formulations tested at visit 1 (high viscosity, intermediate viscosity, and reconstituted) were found to outperform water-reconstituted SPS consistently in all aspects of the taste assessments. Thus, while the physical properties of RDX7675 increase its palatability compared with SPS, optimization of its formulation leads to further improvements. 


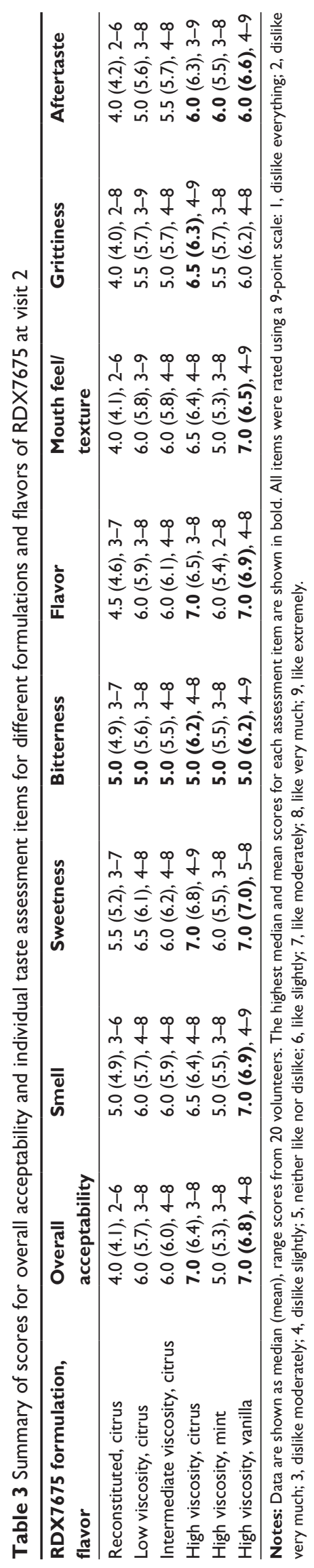

In the palatability testing in healthy volunteers, the high-viscosity preparation was the preferred RDX7675 formulation, with highest or joint highest median scores for all items apart from bitterness and the highest median score for overall acceptability at visit 1 . At visit 2 , the high-viscosity formulations flavored with vanilla or citrus were rated most highly for overall acceptability and between them had the highest or joint highest median scores for all of the individual items. Although the intermediate-viscosity formulation also received high ratings at visit 1 , at visit 2 , participants scored the intermediate-viscosity formulation lower than the vanilla or citrus high-viscosity formulations (except for bitterness, for which all had a median score of 5.0). Generally, for the high-viscosity formulation, vanilla appeared to be the preferred flavor among participants. Although both the vanilla and citrus high-viscosity formulations were highly rated at visit 2 , the vanilla high-viscosity formulation had the highest or joint highest median and mean scores across all items apart from grittiness. Furthermore, slightly more participants rated the high-viscosity citrus formulation unfavorably for overall acceptability compared with the high-viscosity vanilla formulation (three volunteers and one volunteer, respectively).

The palatability of an oral formulation is an important factor influencing how acceptable a treatment is to patients, particularly treatments used for chronic conditions. Acceptability is likely to be of particular importance to patients most at risk of hyperkalemia, such as those with heart failure and/or CKD, who are prescribed multiple medications to take at various times of the day. Currently available sodium and calcium polystyrene sulfonate resins used for the treatment of hyperkalemia are acknowledged to have a gritty texture and unpleasant taste, which can make them difficult for patients to take. ${ }^{23,24}$ Studies involving other oral medications also show the importance of palatability. Unpleasant taste was associated with poor compliance with therapy in patients receiving antibiotics for the treatment of skin and soft tissue infections, ${ }^{25}$ while a survey of elderly patients and their health care professionals showed that compliance with prescribed oral nutritional supplements was low and was affected by taste, texture, and flavor, among other factors. ${ }^{26}$

The issue of palatability, and how this affects patient acceptability and adherence to therapy, is also pertinent to other novel ion exchange treatments developed for hyperkalemia. These include patiromer (Relypsa, Inc., Redwood City, CA, USA), which is approved in the US, ${ }^{27}$ and sodium zirconium cyclosilicate (ZS-9; AstraZeneca Pharmaceuticals LP, Wilmington, DE, USA), for which the marketing 
Increasing citrus-flavored formulation viscosity
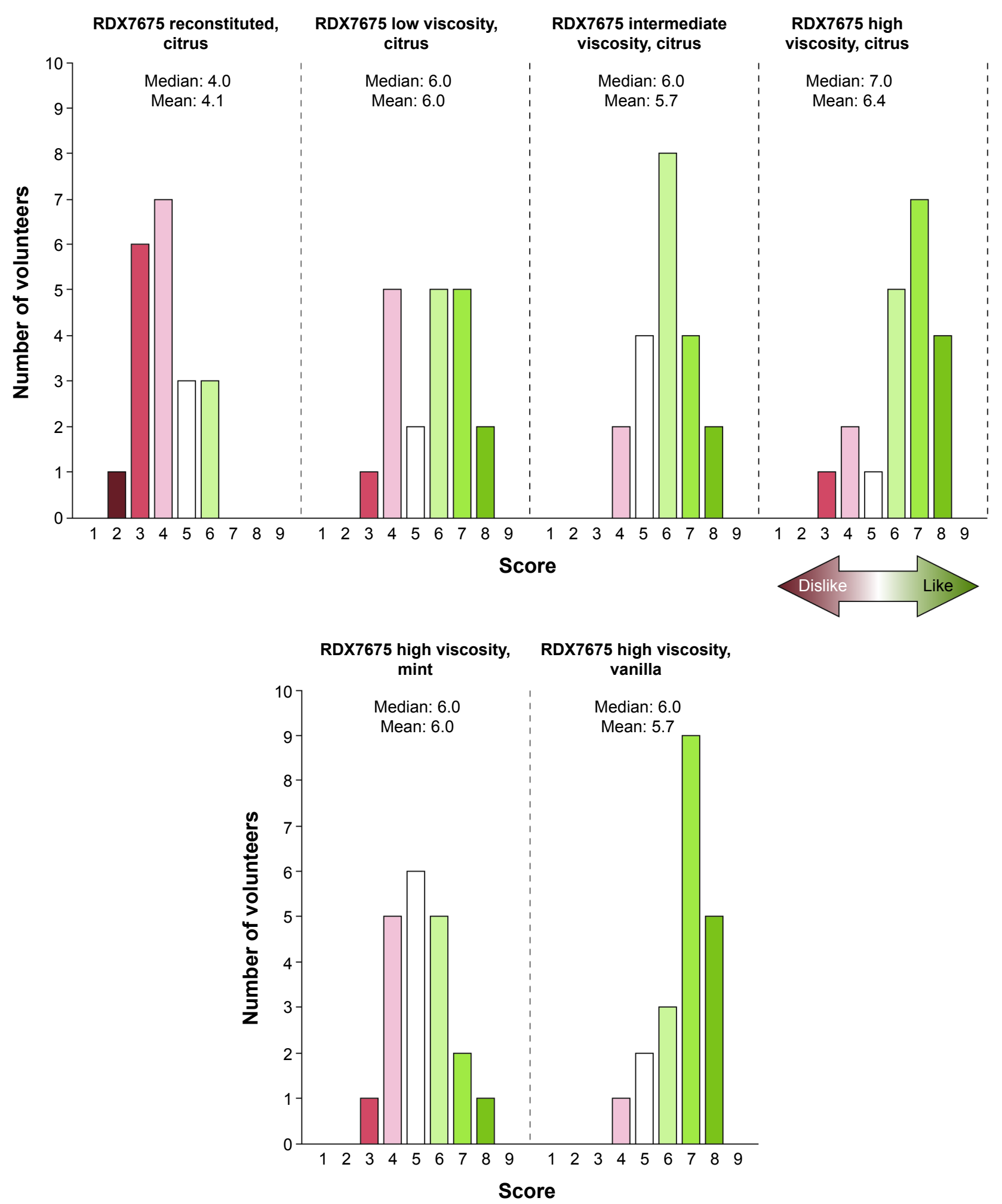

Figure 4 Distribution of scores for overall acceptability of different formulations and flavors of RDX7675 at visit 2.

Notes: Each formulation was rated by 20 healthy volunteers. Overall acceptability was rated on a 9-point scale (I, dislike everything; 2, dislike very much; 3, dislike moderately; 4, dislike slightly; 5 , neither like nor dislike; 6, like slightly; 7, like moderately; 8 , like very much; 9 , like extremely); no participant gave any formulation a score of I or 9. 
application is currently under review in the US. Patiromer has been shown to reduce serum potassium levels in shortterm clinical trials in patients with hyperkalemia and CKD or heart failure, ${ }^{28,29}$ and in a long-term study in individuals with hyperkalemia and diabetic nephropathy. ${ }^{30}$ Like RDX7675, patiromer is an insoluble, non-absorbed polymer made up of spherical particles; approximately $90 \%$ of patiromer particles are in the size range $74-179 \mu \mathrm{m}$, considerably larger than RDX7675. ${ }^{31}$ ZS-9 is an insoluble, inorganic mineral that sequesters potassium ions for excretion in the feces, and like SPS, consists of milled, irregularly shaped particles $(80 \%$ in the range $4-57 \mu \mathrm{m}^{32}$ ). Two large clinical trials have shown that ZS-9 lowers potassium levels in a broad cross section of patients with hyperkalemia. ${ }^{33,34}$ However, palatability studies have not yet been reported for either ZS-9 or patiromer.

\section{Conclusion}

In summary, all formulations of RDX7675 were considered to be markedly more palatable than SPS, including waterreconstituted RDX7675 prepared in the same way as SPS. This demonstrates the positive effect that the characteristics of RDX7675 - smooth, malleable, spherical particles with a narrow size range - have on palatability. Of the different RDX7675 formulations, the high-viscosity formulation was rated most highly by healthy volunteers, with vanilla being the preferred flavor. The overall findings of this study are promising in terms of future patient acceptability of RDX7675 treatment.

\section{Acknowledgments}

The palatability study was performed by Quotient Clinical, Nottingham, UK, and the Principal Investigator was Jo Collier, MBChB, FFPM, Dip Stats. Sensory Research Ltd, Cork, Ireland, developed the taste assessment questionnaire used in the study and provided training on the "sip and spit" technique. Medical writing support was provided by Richard Claes, PhD, and Steven Inglis, PhD, of PharmaGenesis London, London, UK, funded by Ardelyx.

\section{Disclosure}

Vanessa Zann and John McDermott are employees of Quotient Clinical. Jeffrey W Jacobs, James P Davidson, Fangling Lin, Paul Korner, Robert C Blanks, and David P Rosenbaum are employees of Ardelyx and have ownership interest in Ardelyx. The authors report no other conflicts of interest in this work.

\section{References}

1. Dunn JD, Benton WW, Orozco-Torrentera E, Adamson RT. The burden of hyperkalemia in patients with cardiovascular and renal disease. Am J Manag Care. 2015;21(15 Suppl):s307-s315.
2. Khanagavi J, Gupta T, Aronow WS, et al. Hyperkalemia among hospitalized patients and association between duration of hyperkalemia and outcomes. Arch Med Sci. 2014;10(2):251-257.

3. Einhorn LM, Zhan M, Hsu VD, et al. The frequency of hyperkalemia and its significance in chronic kidney disease. Arch Intern Med. 2009; 169(12):1156-1162.

4. Torlen K, Kalantar-Zadeh K, Molnar MZ, Vashistha T, Mehrotra R. Serum potassium and cause-specific mortality in a large peritoneal dialysis cohort. Clin J Am Soc Nephrol. 2012;7(8):1272-1284.

5. Kovesdy CP, Regidor DL, Mehrotra R, et al. Serum and dialysate potassium concentrations and survival in hemodialysis patients. Clin J Am Soc Nephrol. 2007;2(5):999-1007.

6. Jain N, Kotla S, Little BB, et al. Predictors of hyperkalemia and death in patients with cardiac and renal disease. Am J Cardiol. 2012; 109(10):1510-1513.

7. Kovesdy CP. Management of hyperkalemia: an update for the internist. Am J Med. 2015;128(12):1281-1287.

8. Sarafidis PA, Blacklock R, Wood E, et al. Prevalence and factors associated with hyperkalemia in predialysis patients followed in a low-clearance clinic. Clin J Am Soc Nephrol. 2012;7(8):1234-1241.

9. Ben Salem C, Badreddine A, Fathallah N, Slim R, Hmouda H. Druginduced hyperkalemia. Drug Saf. 2014;37(9):677-692.

10. Hollander-Rodriguez JC, Calvert JF Jr. Hyperkalemia. Am Fam Physician. 2006;73(2):283-290.

11. Yancy CW, Jessup M, Bozkurt B, et al. 2013 ACCF/AHA guideline for the management of heart failure: executive summary: a report of the American College of Cardiology Foundation/American Heart Association Task Force on Practice Guidelines. Circulation. 2013;128(16): $1810-1852$.

12. Yancy CW, Jessup M, Bozkurt B, et al. 2016 ACC/AHA/HFSA focused update on new pharmacological therapy for heart failure: an update of the 2013 ACCF/AHA guideline for the management of heart failure. A report of the American College of Cardiology/American Heart Association Task Force on Clinical Practice Guidelines and the Heart Failure Society of America. Circulation. 2016;134(13):e282-e293.

13. National Kidney Foundation Kidney Disease Outcomes Quality Initiative (NKF KDOQI). Clinical practice guidelines on hypertension and antihypertensive agents in chronic kidney disease. Available from: https://www2.kidney.org/professionals/kdoqi/guidelines_bp/. Accessed January 11, 2017.

14. Palmer BF. Managing hyperkalemia caused by inhibitors of the renin-angiotensin-aldosterone system. $N$ Engl J Med. 2004;351(6): 585-592.

15. Weir MR, Rolfe M. Potassium homeostasis and renin-angiotensinaldosterone system inhibitors. Clin J Am Soc Nephrol. 2010;5(3): 531-548.

16. Epstein M, Reaven NL, Funk SE, McGaughey KJ, Oestreicher N, Knispel J. Evaluation of the treatment gap between clinical guidelines and the utilization of renin-angiotensin-aldosterone system inhibitors. Am J Manag Care. 2015;21(11 Suppl):S212-S220.

17. Chaitman M, Dixit D, Bridgeman MB. Potassium-binding agents for the clinical management of hyperkalemia. P T. 2016;41(1):43-50.

18. Krikken JA, Laverman GD, Navis G. Benefits of dietary sodium restriction in the management of chronic kidney disease. Curr Opin Nephrol Hypertens. 2009;18(6):531-538.

19. Rastergar A, Soleimani M. Hypokalaemia and hyperkalaemia. Postgrad Med J. 2001;77(914):759-764.

20. Harel Z, Harel S, Shah PS, Wald R, Perl J, Bell CM. Gastrointestinal adverse events with sodium polystyrene sulfonate (Kayexalate) use: a systematic review. Am J Med. 2013;126(3):264.e9-264.e24.

21. Pongjanyakul T, Priprem A, Chitropas P, Puttipipatkhachorn S. Effect of polysulfonate resins and direct compression fillers on multipleunit sustained-release dextromethorphan resinate tablets. AAPS PharmSciTech. 2005;6(2):E190-E197.

22. Cheng YH, Watts P, Hinchcliffe M, et al. Development of a novel nasal nicotine formulation comprising an optimal pulsatile and sustained plasma nicotine profile for smoking cessation. J Control Release. 2002;79(1-3):243-254. 
23. Schröder CH, van den Berg AM, Willems JL, Monnens LA. Reduction of potassium in drinks by pre-treatment with calcium polystyrene sulphonate. Eur J Pediatr. 1993;152(3):263-264.

24. Tomino Y, Yamazaki T, Shou I, et al. Dose-response to a jelly preparation of calcium polystyrene sulfonate in patients with hyperkalemia changes in serum potassium levels with or without a RAAS inhibitor. Clin Nephrol. 2007;68(6):379-385.

25. O'Donoghue MN. Compliance with antibiotics. Cutis. 2004;73(Suppl 5): 30-32.

26. Lad H, Gott M, Gariballa S. Elderly patients compliance and elderly patients and health professional's, views, and attitudes towards prescribed sip-feed supplements. J Nutr Health Aging. 2005;9(5):310-314.

27. Veltassa (patiromer) for oral suspension [prescribing information]. Redwood City, CA, USA: Relypsa Inc.; 2015. Available from: https:// www.accessdata.fda.gov/drugsatfda_docs/label/2015/205739s000lbl. pdf. Accessed April 13, 2017.

28. Pitt B, Anker SD, Bushinsky DA, Kitzman DW, Zannad F, Huang IZ; PEARL-HF Investigators. Evaluation of the efficacy and safety of RLY5016, a polymeric potassium binder, in a double-blind, placebocontrolled study in patients with chronic heart failure (the PEARL-HF) trial. Eur Heart J. 2011;32(7):820-828.
29. Weir MR, Bakris GL, Bushinsky DA, et al. Patiromer in patients with kidney disease and hyperkalemia receiving RAAS inhibitors. $N$ Engl J Med. 2015;372(3):211-221.

30. Bakris GL, Pitt B, Weir MR, et al; AMETHYST-DN Investigators. Effect of patiromer on serum potassium level in patients with hyperkalemia and diabetic kidney disease: the AMETHYST-DN randomized clinical trial. JAMA. 2015;314(2):151-161.

31. Li L, Harrison SD, Cope MJ, et al. Mechanism of action and pharmacology of patiromer, a nonabsorbed cross-linked polymer that lowers serum potassium concentration in patients with hyperkalemia. J Cardiovasc Pharmacol Ther. 2016;21(5):456-465.

32. Keyser DJ, Guillem AF, inventors; ZS Pharma, Inc., assignee. Microporous zirconium silicate for the treatment of hyperkalemia. United States patent US 20150359821 A1. 2015 Dec 17.

33. Kosiborod M, Rasmussen HS, Lavin P, et al. Effect of sodium zirconium cyclosilicate on potassium lowering for 28 days among outpatients with hyperkalemia: the HARMONIZE randomized clinical trial. JAMA. 2014;312(21):2223-2233.

34. Packham DK, Rasmussen HS, Lavin PT, et al. Sodium zirconium cyclosilicate in hyperkalemia. N Engl J Med. 2015;372(3):222-231.

\section{Publish your work in this journal}

Drug Design, Development and Therapy is an international, peerreviewed open-access journal that spans the spectrum of drug design and development through to clinical applications. Clinical outcomes, patient safety, and programs for the development and effective, safe, and sustained use of medicines are the features of the journal, which

\section{Dovepress}

has also been accepted for indexing on PubMed Central. The manuscript management system is completely online and includes a very quick and fair peer-review system, which is all easy to use. Visit http://www.dovepress.com/testimonials.php to read real quotes from published authors.

Submit your manuscript here: http://www.dovepress.com/drug-design-development-and-therapy-journal 